\title{
NOTAS SOBRE DISCRECIONALIDAD Y LEGITIMACIÓN
}

\section{El lugar de la discrecionalidad en un Derecho en cambio}

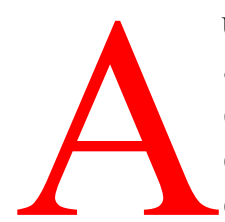

unque me hubiera gustado hablar de cuestiones de legitimidad, porque creo que afectan al núcleo de los problemas de justificación y exige un gran esfuerzo en torno a la construcción de criterios de racionalidad y justicia -legitimidad crítica- tal como hace Elías Díaz, mi propósito es mucho más modesto. Se circunscribe principalmente al área de la legitimación, a la percepción por parte de los ciudadanos de la conexión entre Derecho, ejercicio del poder y criterios de justificación en un momento en el que sobre el Derecho planean importantes transformaciones sociales. Entre todos los aspectos que cabría analizar mi interés se encuentra también limitado a una sola de las cuestiones que es posible suscitar en este contexto: el alcance de la discrecionalidad. Es cierto, que la existencia de decisiones discrecionales o de lo que se denomina un «Derecho flexible» no es un fenómeno enteramente novedoso, sin embargo, los cambios operados en muchos sistemas jurídicos han hecho de la discrecionalidad un factor suficientemente central como para que repercuta en algunas de las proyecciones de la legitimación del sistema.

Como resultaría imposible en este trabajo tratar de explicar los cambios a los que me he referido y sobre todo describir y calibrar adecuadamente sus causas, fijaré mi atención sólo en algunos aspectos muy concretos de las formas de regulación que el poder político ha elegido para ejercer el poder a través del Derecho, y aquí nos encontramos con aspectos que aunque puedan parecer de matiz, en mi opinión constituyen un indicador de algunas de las direcciones que hoy adopta el Derecho y que están relacionadas con su legitimación. De lo que no es posible desentenderse, sin embargo, es de que el objeto de análisis -el Derecho- pasa por momentos de cambio o, 
dicho con otros términos, precisa acomodarse a nuevas formas y a funciones distintas, lo que exige, como consecuencia, nuevas categorías, conceptos e instituciones que comienzan a primar frente a otras que han sido tradicionalmente básicas.

En este sentido, quisiera llamar la atención sobre dos perspectivas que a la vez están relacionadas. De un lado, que los cambios y transformaciones que se están produciendo en el orden social, económico, cultural, exigen que el Estado y el Derecho desempeñen nuevas funciones. Sin embargo, si se observa cómo se está articulando este proceso, vemos que no consiste tanto en sustituir funciones tradicionales o clásicas por otras, sino en aumentar o solapar nuevas dimensiones funcionales, en algunos casos yuxtaponiendo unas a otras. Lo que, claro está, no puede producirse sin tensiones y contradicciones. Así, tradicionalmente, el Estado ha desempeñado, entre otras, una función de tratamiento de conflictos y de orientación social a las que después se unieron tareas de prestación de servicios públicos y de intervención económica; ahora además asume un cierto papel de intermediario o moderador, de organizador y coordinador de objetivos sociales y de intereses públicos y privados, que ha dado lugar a una importante descentralización de las decisiones, a que los procedimientos negociadores ocupen un lugar destacado y no periférico y a mayores grados de flexibilidad. Lo que comporta, desde el punto de vista jurídico, nuevos problemas de regulación, procedimientos de toma de decisiones, estructuras y acciones que tienen unas facetas distintas ${ }^{1}$. Pero además, esas modificaciones tienen efectos en la percepción que los ciudadanos tienen del Estado y del Derecho y en sus expectativas y exigencias. Uno de los problemas que surgen desde estos presupuestos es si los nuevos modos de ejercicio del poder estatal se establecen sobre la base de la aceptación pública del poder político, es decir, de su legitimación.

De otro lado, y como es sabido, en el orden jurídico nos encontramos, por un conjunto de causas y factores, entre las que se encontrarían las que he mencionado, con un cambio en el centro de gravedad del mismo que hoy se sitúa básicamente en la toma de decisiones y en su justificación. Por tanto el cambio de funciones del Derecho se produce íntimamente conectado a la expresión de nuevas concepciones sobre el Derecho y a una distribución distinta de papeles entre los operadores jurídicos. Si la toma de decisiones pasa a

\footnotetext{
${ }^{1}$ Sobre los factores, procesos y consecuencias de los cambios sociales de los que he hecho mención existe una abundante bibliografía imposible de recoger aquí. No obstante y concretamente por lo que respecta a las repercusiones que pueden tener en el Derecho me parece muy interesante la lectura del primer capítulo del libro de L. Parejo, Crisis y renovación en el Derecho Público, Madrid, Centro de Estudios Constitucionales, 1991.
} 
ocupar un lugar central significa que cobran una relevancia especial todos los titulares de ese poder de decisión, especialmente claro está legislador y juez y en segundo lugar otros muchos operadores jurídicos. En este sentido no es posible desconocer que los sistemas jurídicos se caracterizan en la actualidad porque en ellos se produce una dispersión del poder de toma de decisiones. Entre otras razones porque su estructura está cada vez más descentralizada y, por otro lado, la actividad interpretativa cobra un valor especial. Por otra parte, y desde la perspectiva de la decisión, se hace especial hincapié en la diversidad de razones que es posible adoptar a la hora de motivar una decisión jurídica, porque entre ellas no interesan sólo las normas jurídicas válidas asumidas como premisas, ni las proposiciones descriptivas de hechos, sino también diversos argumentos que van dirigidos a justificar la aceptación de ciertas normas o los que están orientados a la toma en consideración de las consecuencias de una decisión o a los fines y objetivos que se pretende realizar. Todo ello, como veremos tiene una proyección importantísima en la discrecionalidad.

El Derecho, como orden normativo predominante en una sociedad y monopolizado por el Estado, ha sido el fundamento y el instrumento de poder estatal moderno; modelo de Estado basado en la concepción del Estado de Derecho, es decir, en la idea de un poder político sometido a normas que él mismo produce. En este sentido, el Derecho ha sido considerado tanto como un instrumento del Estado, como factor elemental de legitimación del mismo y esta decisiva función, entre otras, como tantas veces ha subrayado Elías Díaz ${ }^{2}$, es la que hace del Derecho una realidad suficientemente autónoma, capaz de manifestar una influencia propia y específica en el grupo social y, por tanto, su aceptación y su efectividad devienen de su congruencia con valores, intereses, usos sociales o tensiones del grupo social y no de la concentración del poder político en manos del Estado.

Como tantas veces se ha señalado de un tiempo a esta parte, el Estado de corte europeo occidental, como tipo de Estado, se encuentra

2 Elías Díaz, Sociología y Filosofía del Derecho, Madrid, Taurus, (1971) 1989, págs. 11-12, 201-204. A estos efectos, habría que llamar la atención sobre el punto de vista de las teorías «sistémicas» que si bien aciertan a explicar el sistema social como conjunto de dimensiones funcionales o subsistemas que se encuentran interrelacionados, sin embargo, tienen que hacer frente a importantes obstáculos a la hora de explicar qué subsistema tiene, en cada momento, mayor capacidad para estructurar las formaciones sociales. Ciertamente, todos tienen relaciones de dependencia respecto de los demás, pero esto no puede significar que se encuentren en planos de igualdad; de lo contrario no se podría explicar, por ejemplo, cuáles son las razones por las cuales el subsistema político muestra una capacidad limitada para dirigir la economía. 
sometido a un intenso debate y además su propia existencia está permeada de importantes tensiones y movimientos contradictorios o paradójicos, sometido aun a la inercia de su propia evolución ${ }^{3}$. Como señala Parejo Alfonso $^{4}$ el éxito del modelo socioeconómico y político que se va afianzando a través de la fórmula del Estado Social de Derecho genera nuevas condiciones sociales, económicas y culturales que acaban por desbordar el marco de referencia que las hacía posibles. La quiebra del principio del crecimiento y desarrollo continuados y los límites de la expansión de las funciones redistributivas e integradoras del poder público minan la confianza en la capacidad del Estado para la dirección efectiva de los procesos sociales. Pero esta quiebra, como escribe Elías Díaz ${ }^{5}$, no lo es sólo de unas condiciones y un modelo económico, sino también una crisis de legitimación, es decir, de valores culturales y sociales, de concepciones del mundo que la sociedad expresa, también, contradictoriamente y donde conviven valores marcadamente individualistas con perspectivas que subrayan la dependencia social del individuo en un marco social complejo en el que la interrelación social y la contradicción entre los diversos intereses se hace cada vez más patente. Todo ello, además cuando la construcción jurídica del Estado y del Derecho público, desde el punto de vista dogmático, aun pervive solapando principios, categorías y técnicas procedentes tanto del Estado Liberal de Derecho como del Estado Social ${ }^{6}$. La yuxtaposición de dimensiones,

\footnotetext{
${ }^{3}$ Entre otras razones, por la falta de claridad de la propia definición de poder público, identificada reductivamente en determinados momentos con la idea de poder estatal y depositada en la actualidad en manos tanto de poderes públicos como privados que imponene su lógica y su protagonismo a partir de parámetros de racionalidad limitados, en muchas ocasiones a postulados de eficiencia que plantean importantes problemas respecto de su legitimidad. J. J. Aguirre de la Hoz, La dicotomía público-privado: descripción y crítica. Ideología e historia de su constitución, Tesis Doctoral, Dirigida por J. de Lucas, Valencia, 1993, pág. 181-182. 652, 669-677, 741, 753-754. Vid. también, Olivas, «Problemas de legitimación del Estado social», cit. págs. 25-25.

${ }^{4}$ L. Parejo Alfonso, Crisis y renovación en el Derecho Público, op. cit., págs. 11-13.

${ }^{5}$ Elías Díaz, «El nuevo contrato social: instituciones políticas y movimientos sociales», Crisis y futuro del estado de bienestar, R. Muñoz de Bustillo (comp), Madrid, Alianza, pág. 227.

${ }^{6}$ A esta dualidad de la regulación jurídica del Estado hacen referencia muchos autores a través de la referencia a realidades ya acuñadas a las que se refieren como «Derecho informal», Infra droit, etc.. En nuestro contexto podemos referirnos a J. A. Santamaría Pastor que habla de tres niveles o esferas en el Derecho administrativo. Un derecho oficial, cuyos principios se recogen en la Constitución y en las principales leyes, pero cuya estructura responde fundamentalmente a la del Estado liberal. Un derecho subterráneo que ha afectado directamente al principio de legalidad y a la teoría de fuentes y finalmente, un derecho marginal que se ha desarrollado al margen
} 
a veces, forzada y contradictoria de los dos modelos de Estado ha conducido a una combinación compleja entre funciones de control de orden jurídico, político y social ${ }^{7}$. La evolución del Estado social ha dado lugar a dos dimensiones fundamentales de este modelo de Estado de Derecho, la que se denomina materialización del Derecho que, como muestra Estévez $^{8}$, es una consecuencia de la puesta en cuestión de determinados presupuestos del diseño del Estado liberal clásico. En particular, de las relaciones entre política y economía y de la división de poderes. Y una segunda línea evolutiva que se denomina procedimentalización del Derecho, a través de la cual, el Derecho desempeña una función facilitadora que se desarrolla básicamente a través de la regulación de procedimientos, formas de participación y organización, cuyo objeto es lograr una cierta autorregulación de diverso sectores jurídicos ${ }^{9}$.

\section{Discrecionalidad: alcance y límites}

La diversificación de funciones del Derecho, su articulación como un complejo policéntrico de titulares de decisión, las exigencias que provienen del carácter central de la toma de decisiones, el tipo de procedimientos que comportan y el papel de los operadores jurídicos, han contribuido, entre otros factores a dar un mayor peso a la discrecionalidad en el orden jurídico. La idea que vertebra un proceso así es que la distribución de poderes de decisión se atribuye a instancias que por sus rasgos característicos reúnen mejores condiciones para adoptar una decisión lo más acertada posible.

La discrecionalidad ha sido calificada como un aspecto central del Derecho y a la vez inevitable ${ }^{10}$. Central porque los sistemas jurídicos

${ }^{7}$ E. Olivas, «Problemas de legitimación en el Estado social», en Problemas de legitimación en el Estado social, Madrid, Trotta, 1991, págs. 12-13.

${ }^{8}$ J. A. Estévez Araujo, «Estructura y límites del Derecho como instrumento del Estado social» en Problemas de legitimación en el Estado social, op. cit. págs. 151-155 y 157-158.

${ }^{9}$ G. Teubner, «After Legal Instrumentalism? Strategic models of postregulatory Law», Dilemas of Law in the Welfare State, Berlín, New York, Walter de Gruyter, 1985, págs. 314-319. Donde explica las transformaciones en las relaciones entre Derecho y poder y la tendencia a la delegación de competencia para la toma de decisiones hacia organizaciones privadas y público-privadas que, como otra cara de la moneda, ponen de relieve que esas capacidades de decisión hacen de las instituciones entidades políticamente responsables. esp. págs. 317-318.

${ }^{10} \mathrm{~K}$. Hawkins, «The Use of Legal Discretion: Perspectives from Law and 
contemporáneos distribuyen cada vez más poderes expresos de decisión a funcionarios y operadores en general con objeto de realizar los objetivos legislativos de carácter más general ${ }^{11}$. Inevitable por que el paso de las normas a la acción, de la abstracción a la realidad, implica sujetos que interpretan, realizan y adoptan decisiones. Este último aspecto encierra la perspectiva más tradicional sobre la discrecionalidad, es decir, el planteamiento sobre el alcance de decisiones individuales que proporcionan las normas y es lo que ha llevado a pensar, durante mucho tiempo, que la discrecionalidad no es sino algo periférico en relación con un núcleo de normas que es el que da entidad a un sistema jurídico. Tal punto de vista es, con palabras de Galligan ${ }^{12}$ reductivo, menosprecia la discrecionalidad como una desviación y no alcanza a la dimensión más importante de la discrecionalidad que se encuentra precisamente en el modo de ejercicio del poder y su legitimidad, es decir, en todas aquellas áreas en las que se confiere un poder de decisión y donde en mayor o menor medida ese poder puede determinar también los criterios de acuerdo con los cuales se ejerce un abanico de competencias. Por otra parte, ha ocurrido también que allí donde se ha prestado mayor atención a la discrecionalidad dentro del orden jurídico, se ha centrado la atención en el razonamiento judicial de los tribunales superiores, especialmente, en la forma en que los jueces justifican sus decisiones en los casos difíciles y no se ha prestado tanta atención a la toma de decisiones de funcionarios judiciales de menor rango o de los funcionarios de la administración. Con todo, es muy importante recordar que la presencia creciente de discrecionalidad ni proporciona inmunidad ni es equivalente a una toma de decisiones arbitraria, por el contrario, su existencia, correlativa, al cambio de funciones operado por muchos sistemas jurídicos es, antes que nada, un desafío para la existencia de controles jurídicos y sociales críticos e inescindible de la exigencia de mayores cuotas de responsabilidad por quiénes son titulares de un poder de decisión ${ }^{13}$.

Social Science», The Uses of Discretion, Oxford, Oxford University Press, 1992, pág. 11.

${ }^{11}$ El punto de vista clásico de los teóricos del Derecho sobre la discrecionalidad centra su atención en el Derecho como sistema de normas y criterios que en cierto modo predeterminan la decisión. En todo caso, el comportamiento discrecional quedaría limitado a los casos de ausencia o indeterminación del material jurídico. Es decir, se plantearía como uno de los problemas que genera la interpretación jurídica en el supuesto de falta de claridad o en los casos de lagunas.

${ }^{12}$ D. J. Galligan, Discretionary Powers. A Legal Study of Official Discretion, Oxford, Clarendon Press, 1986, págs. 1-2.

${ }^{13}$ Es bien conocida la dicotomía que expresa A. V. Dicey al contrastar un sistema de autoridad donde los poderes de los funcionados están regulados por normas jurídicas generales y un sistema en el que los poderes son «amplios, arbitrarios o 
Ciertamente, el punto de vista que se adopta en relación a la discrecionalidad es plural y depende básicamente de la visión que se tiene del Derecho y de la función de los operadores en él. Como tantas veces se ha escrito ${ }^{14}$, el viejo anhelo del formalismo jurídico -plenitud del ordenamiento jurídico, carácter mecánico del proceso de aplicación, única respuesta correcta, etc- ha sido fuertemente criticado, y sin embargo, sus premisas ideológicas han pervivido inalteradas durante mucho tiempo y defendidas en la actualidad por posiciones enfrentadas al positivismo como es, por ejemplo, la representada por Dworkin $^{15}$. Desde estos presupuestos el papel de los operadores jurídicos y especialmente de los jueces está estrechamente unido a los presupuestos ideológicos a los que me refería. De tal forma que la pervivencia de un modelo de juez que debe encontrar siempre la respuesta correcta porque ésta existe en el orden jurídico

discrecionales», The Law of the Constitution, London, MacMillan, 1961, pág. 188. Es característico de la utilización del término en el lenguaje jurídico que una decisión es arbitraria no cuando es menos racional de lo que podría ser, sino cuando no alcanza el mínimo umbral de racionalidad. K. Hawkins indica también, a estos efectos, cómo la acusación de arbitrariedad o del carácter caprichoso de una decisión surge de la distancia que se produce entre las expectativas que produce la toma en consideración de una norma jurídica y cómo se debe adoptar una decisión y de otro lado, las determinaciones sociales prácticas de las decisiones y los modelos de comportamiento rutinario de los operadores. «The Uses of Discretion», cit. pág. 13.

${ }^{14}$ Por lo que respecta al planteamiento de la tensión entre unidad de solución justa y discrecionalidad, vid. L. Prieto, Sobre principios y normas. Problemas del razonamiento jurídico, Madrid, Centro de Estudios Constitucionales, 1992, págs, 103 y ss, 119-120.

${ }^{15}$ R. Dworkin en su obra Los derechos en serio, Barcelona, Ariel, 1984, trad. M. Guastavino, establece, como es sabido, la distinción entre un sentido débil de término discrecionalidad y un sentido fuerte. El primer sentido hace referencia a una decisión que se basa en una norma que ha de ser interpretada, que no admite una aplicación mecánica o bien cuando una autoridad adopta una decisión que no es susceptible de ser revisada por ninguna instancia superior (pág. 49). Habla de discrecionalidad en sentido fuerte para aquellos casos en que el juzgador no está vinculado por criterios impuestos por la autoridad y debe crear su propio criterio de decisión (pág. 85). Su concepción integrativa del Derecho, la confianza que muestra hacia la unidad de solución justa, la estructura del razonamiento judicial que defiende y la posición institucional de quien administra justicia le llevan, como indica L. Prieto en Sobre principios y normas, cit. págs. 111 y ss. a excluir la discrecionalidad fuerte, que se limitaría a los supuestos de lagunas y de soluciones normativas injustas o inadecuadas a la luz de los principios- y a reducir el peso de la discrecionalidad débil que se resuelve a través del ejercicio del discernimiento o buen sentido, porque en el fondo no acepta las consecuencias de este tipo de discrecionalidad (pág. 115). En definitiva, «para Dworkin la discrecionalidad no es aceptable en ningún sentido porque a la luz del Derecho -tal y como él lo entiende- debe existir siempre una respuesta correcta $\mathrm{o}$, al menos, una más correcta que cualquier otra» (pág. 116). 
preconstituida a la decisión del caso $^{16}$, ha comportado el mantenimiento de un juez irresponsable o, al menos, aunque sea responsable desde el punto de vista técnico, no lo es desde el punto de vista político porque su decisión está determinada previamente.

Dworkin, sin embargo, tiene razón cuando afirma que la discrecionalidad sólo cobra sentido en contextos normativos, «El concepto de discrecionalidad -escribe- sólo es adecuado en un único tipo de contexto; cuando alguien está en general encargado de tomar decisiones sujetas a las normas establecidas por una autoridad determinada» ${ }^{17}$. Es un problema que se plantea inmediatamente al adentrarse en la complejidad de los procesos y en la diversidad de sujetos que adoptan decisiones. Es decir, con Galligan ${ }^{18}$, la discrecionalidad no es tanto un efecto colateral de la existencia de normas, sino un modo de conferir poderes allí donde se considera importante que los operadores y especialmente los funcionarios adopten decisiones atendiendo fundamentalmente a los criterios que deben guiar su forma de ejercer el poder. Normas y principios no son el reverso de la discrecionalidad, puesto que son ellos los que constituyen, definen y someten a sujeción la discrecionalidad. Para K. Hawkins no es sino «el espacio entre las normas jurídicas en el que los operadores pueden ejercer su capacidad de decisión ${ }^{19}$. Sin embargo, es importante tener en cuenta que el alcance y el contexto en el que se ejerce la discrecionalidad no afecta sólo a sus límites jurídicos y a su definición, porque en primer lugar es un ejercicio de poder social y en ese sentido en ella se producen importantes implicaciones entre valores sociales y jurídicos ${ }^{20}$.

${ }^{16}$ Entre otros muchos textos, para un examen sintético de las diferentes versiones de la tesis de la respuesta correcta, puede verse A. Aarnio, «La tesis de la única respuesta correcta y el principio del razonamiento jurídico», Doxa, $\mathrm{n}^{\circ} .8$, págs. 24, 32 ss.

${ }^{17}$ R. Dworkin, Los derechos en serio, op. cit. págs. 84-85.

${ }^{18}$ D. J. Galligan, Discretionary Powers, op, cit. pág. 2. Donde insiste en que la discrecionalidad del poder no es un fenómeno meramente periférico al ejercicio de autoridad, ni tampoco una desviación indeseable de un ideal de gobierno a través de normas.

${ }^{19} \mathrm{~K}$. Hawkins, «The Uses of Discretion», cit. pág. 11.

${ }^{20}$ De entre el abanico de cuestiones que plantea la discrecionalidad este es uno de los más importantes. Al respecto puede verse, por ejemplo, el trabajo de John Bell, «Discretionary Decision-Making: A Jurisprudential View», en The Uses of Discretion, op. cit. págs. 95 y ss. Sobre ello inciden muchos autores y es importante destacar que, en este sentido, el punto de vista de los científicos sociales es quizá más amplio que el de los teóricos del Derecho, porque sitúan el análisis de la discrecionalidad en el contexto general de la toma de decisiones Y prestan atención a los condicionamientos de tipo social, económico y político que afectan al ejercicio del poder. 
En el tratamiento doctrinal sobre la discrecionalidad suele señalarse dos aspectos o dimensiones de la misma. Por un lado el relativo a la elección y por otro el del poder. Por lo que respecta al primer aspecto, casi todas las definiciones de discrecionalidad comienzan con la noción de elección ${ }^{21}$. En este sentido, un poder discrecional es entendido como capacidad de decidir circunscrita a un conjunto de límites. La idea de elección, por su parte, como indica Venezia, hace referencia al grado de autodeterminación que posee el sujeto que decide y al área sobre la que ejerce la elección, como una consecuencia de la responsabilidad que tiene. La elección que realiza el operador jurídico puede afectar a la decisión sobre si debe actuar, sobre cómo debe hacerlo y sobre las razones apropiadas para su acción ${ }^{22}$. El ejercicio de la discrecionalidad como posibilidad de elegir criterios de decisión, por su parte, no es incompatible, evidentemente, con la existencia de condicionamientos o restricciones, que no quedan limitados a los objetivos que deben ser perseguidos y al espacio en el que se puede elegir, aunque estos criterios cumplen un papel básico en la decisión ya que los términos del poder delegado constituyen, como se ha escrito, las fuentes primarias de orientación, sino que afectan también a la racionalidad en el ejercicio de la discrecionalidad. De modo que la decisión debe resultar justificable desde el punto de vista racional y desde los objetivos que se persiguen al conferir un poder de decisión. Es decir, dentro de la esfera definida de poder, el operador que adopta una decisión debe reflejar los objetivos y propósitos que la guían y establecer los criterios para su realización y, es posible que la discrecionalidad se de en alguno de esos dos factores ${ }^{23}$.

${ }^{21}$ Como recuerda John Bell, tanto en las definiciones que proporcionan Galligan o J. C. Venezia, Le Pouvoir discrétionnaire, París, L. G. D. J., 1959, pág. 132 que son consideradas como los trabajos más importantes de teoría del Derecho sobre la discrecionalidad, como en la contribución de K. C. Davis, Discretionary Justice: A Preliminary Inquiry, Baton Rouge. Louisiana Univ. Press, 1969, pág. 4, se pone el peso del concepto en la comprensión de la discrecionalidad como elección.

${ }^{22}$ Galligan afirma que el sentido básico de la idea de discrecionalidad hace referencia a «un alcance significativo para establecer las razones y estandars de acuerdo con los cuales... se va a ejercer un poder, y para aplicarlas en la toma de una decisión específica», Discretionary Powers, op. cit. pág. 21.

${ }^{23}$ D. J. Galligan señala a estos efectos que los poderes discrecionales pueden ser entendidos como subsistemas de autoridad dentro de los cuales los funcionados tienen diversos grados de libertad y autonomía. Por tanto, que pueden decidir en algún sentido significativo cuáles son los estandars y pautas, así como las estrategias para su realización y su aplicación a casos específicos, sujetos a una guía que deriva de la red de principios que envuelven a la propia institución. Desde este punto de vista, para entender la idea de discrecionalidad hay que poner en relación dos conjuntos de variables. En primer lugar está en función de la presencia relativa o la ausencia de criterios para la decisión, del alcance que pueda tener la valoración por estandars para la decisión, del alcance que pueda tener la valoración y el juicio por 
En este sentido la revisión judicial de las decisiones está estrechamente vinculada a criterios de racionalidad orientados a fines u objetivos. Es posible detectar, a estos efectos, una tendencia a desarrollar criterios generales en la toma de decisiones, que se centran en la definición de objetivos sociales y en criterios sobre las formas de ejercicio del poder lo que da como resultado la formulación de una red de estandars generales, como pautas de acción racional que proporcionan consistencia entre las decisiones. En este sentido, las directrices políticas o las consideraciones sobre el bienestar general (policies) forman una parte muy importante de esta red de criterios $^{24}$. Finalmente, existen también límites institucionales para toda actuación discrecional, ya que los poderes se confieren en un marco institucional que provee el contexto y los límites para la toma de decisiones. De modo que el espacio real de libertad para elegir sólo puede ser valorado adecuadamente desde un marco institucional concreto $^{25}$.

Si la elección implica un grado de autodeterminación en el marco de un conjunto de límites, la discrecionalidad puede producirse, pero también es más amplia que el juicio que se emite en la interpretación y aplicación de criterios. Como indica Bell ${ }^{26}$, tener una libertad

parte de quién decide y de los términos de su autoridad. El segundo conjunto de variables estaría integrado por el punto de vista interno, es decir, por las actitudes de los funcionarios respecto de sí mismos y por los acuerdos institucionales existentes.

${ }^{24}$ Como subraya L. Prieto en relación con las decisiones judiciales, de un lado, la toma en consideración de objetivos sociales no es una práctica judicial antidemocrática, sino que representa un cabal sometimiento a las decisiones constitucionales. De otro, la evaluación de las consecuencias de la decisión, si bien no puede elevarse a único criterio justificatorio, está integrada en el conjunto de criterios generales, en la medida que supone tomar en cuenta el posible impacto de la decisión y tiene una relación directa con funciones básicas del Derecho como es la de estimular o desalentar conductas. Principios y normas, op. cit. págs. 120-121. Con relación al peso de los argumentos de tipo consecuencialista, el autor remite a las tesis de N. Maccormick, «On Legal decisions and their Consequences: from Dewwy to Dworkin», New York University Lav Review, nº. 58, 1983, págs. 240 y ss.

${ }^{25}$ En este sentido habría que tomar en consideración un conjunto relativamente amplio de límites normativos. Como pone de relieve K, Hawkins, la discrecionalidad está ampliamente relacionada con la utilización de normas y con la decisión sobre la relevancia del uso de las normas. Puede ocurrir que desde el punto de vista jurídico una decisión parezca discrecional y flexible y sin embargo. esté orientada y condicionada por normas pero que no son jurídicas en sentido técnico, sino institucionales, sociales o de organización. «The Use of Legal Discretion...», cit. pág. 13. Vid. También, M. Baumgartner, «The Myth of Discretion», págs. 152-156. Otros autores, insisten, sin embargo, en las restricciones que imponen al ejercicio de la discrecionalidad factores tales como la formación de los juristas, la estructura institucional y la dinámica interna de la entidad en la que se encuentra incardinado quién decide. Puede verse, entre otros, C. E. Sscheider, «Discretion and Rules. Lawyer's View», en The Uses of Discretion, op. cit. pág. 79-87.

${ }^{26} \mathrm{~J}$. Bell «Discretionary Decision-Making...», cit. pág. 94. 
genuina de acción dentro de un abanico significativo de opciones es suficiente para identificar la importancia de un poder, desde el punto de vista político, aunque, evidentemente, no se trate de una elección ilimitada. En un sentido estricto, habría discrecionalidad en aquellas decisiones en las que los criterios y su aplicación a casos particulares plantean problemas de determinación final por la propia autoridad delegada. Este planteamiento se reconoce frecuentemente en muchos sistemas jurídicos, pero esta visión de la discrecionalidad no ofrece con carácter previo una defición clara de una forma de poder, por el contrario, existen grados y diversas formas atendiendo a los factores que intervienen en la discrecionalidad ${ }^{27}$. Además los poderes discrecionales, ni siquiera en sentido estricto son inmunes al control jurídico, no sólo porque en última instancia son los tribunales los que determinan, desde un punto de vista jurídico, qué titulares de un poder tienen discrecionalidad y en qué medida sino porque además -escribe Galligan- «pueden constituir un potencial considerable para un sistema de controles jurídicos más crítico y penetrante de los poderes discrecionales ${ }^{28}$.

Una segunda dimensión o aspecto relevante de la idea de discrecionalidad es la existencia de una autorización para determinar los propios criterios de acción por parte de un sujeto en relación a otros. A estos efectos podríamos afirmar que diferentes perspectivas doctrinales dirigen su atención a problemas como las formas a través de las cuales se ejerce el poder, los modos de control del mismo o la forma en que se distribuye el poder en una sociedad. Ciertamente, el

${ }^{27}$ A pesar de la gradación y la limitación de un poder discrecional «auténtico» a estos supuestos, es imposible desconocer, como ponen de relieve numerosos autores la persistente asociación que se produce entre discrecionalidad y discriminación. Cuando se utiliza para justificar mecanismos de exclusión, como una faceta más del ejercicio del poder que no opera sólo sobre quienes toman parte en las decisiones, sino que opera excluyendo a personas y asuntos. Sobre este punto vid. J. Handler, «Discretion: Power, Quiescence, and Trust» en The Uses of Discretion, op. cit. págs. 335 y ss, que remite su examen de las formas de poder a las tesis de S. Lukes, Power, New York, New York University Press, 1986. Uno de los efectos más importantes de la carga negativa que comporta la discrecionalidad, como muestra J. de Lucas, a propósito de la aplicación del principio de igualdad y la condición de ciudadano, se encuentra en hacer de los derechos fundamentales un problema de «administración o de policía» que conduce a través de la actividad interpretativa a una dinámica de fragmentación y división de sujetos. El desafio de las fronteras. Derechos humanos y xenofobia frente a una sociedad plural, Madrid, Temas de Hoy, 1994, págs. 185-197.

${ }^{28}$ D. J. Galligan, Discretionary Powers, op. cit. pág. 23 y 86-87. Es interesante la reflexión del autor sobre los diversos sentidos en los que puede establecerse la relación entre la revisión jurisdiccional de la discrecionalidad y los principios democráticos, así como los cambios apreciables en las actitudes de los jueces, al respecto vid. págs. 234-257. 
modelo o el supuesto básico de poder en el que se produce la discrecionalidad es el que tiene lugar entre funcionarios y ciudadanos, pero también tiene importantes dimensiones en el espacio de las relaciones jurídico-privadas. Aunque, ciertamente, las dimensiones de poder varían de acuerdo con los dos grandes modelos que representan el Derecho público y el privado, a pesar de que hoy una de las transformaciones del Derecho a la que estamos asistiendo es consecuencia del desdibujamiento o la pérdida de límites precisos entre lo público y lo privado en las relaciones sociales. El Derecho privado desempeña una función básicamente facilitadora, esto es, el Derecho tiene la función de proveer condiciones estables para la relaciones privadas en las que las acciones ejecutivas están limitadas a la realización de normas. Aquí la discrecionalidad tiene un papel limitado y lucha por realizar, por obtener un status de legitimidad. Por su parte el modelo de Derecho público el orden social, precisa medidas de intervención y mediación muy importantes, y es aquí donde la discrecionalidad puede entenderse como una parte de la realización de los objetivos políticos, en el sentido más amplio del término, y de este modo plantea mayores problemas en el orden de la legitimación tanto para el sistema político como para el jurídico. Sin embargo, si bien el poder discrecional se muestra preferentemente en la capacidad de decisión de algunos funcionarios esto no significa que la discrecionalidad se produzca sólo en la esfera de acción estatal, por el contrario otra de las manifestaciones importantes de los cambios sociales se sitúa en la expansión del alcance de la discrecionalidad privada por ejemplo, en las áreas que están sometidas en la actualidad a procesos de desregulación. Y no está muy claro que el tipo de discrecionalidad que se produce en estos ámbitos sea de un tipo diferente a la que se ejerce hoy desde los poderes públicos ${ }^{29}$, pero el problema más que en la propia identificación de la discrecionalidad se encuentra, si atendemos a las actitudes de los funcionarios y de todos aquellos que ejercen un poder discrecional, es si coinciden en sus propósitos, esto es en realizar objetivos de bienestar social -Social policies-.

Es decir, la cercanía de los modelos correspondientes al Derecho público y al privado se explica, en parte, de un lado, porque la discrecionalidad no puede ser entendida como una relación de poder unilateral y, de otro porque la descentralización, coordinación interna y separación funcional son rasgos que permean todo el orden jurídico. Así, como muestra L. Parejo ${ }^{30}$, el poder público necesita apoyarse en la esfera de la economía privada y acudir a fórmulas de

${ }^{29}$ J. Bell, «Discretionary Decision-Making...», cit., pág. 96.

${ }^{30}$ L. Parejo Alfonso, Crisis y renovación en el derecho público, op. cit. pág. 20-21. 
concierto, transacción y cooperación con grupos y agentes privados, incluso, como ya he señalado para definir y dar contenido a la categoría de interés público. Este proceso ha dado lugar a la aparición de lo que se denomina «Derecho negociado», para hacer referencia a la idea de que tanto la función de tratamiento de conflictos como la de promoción de las condiciones básicas de la vida social se realizan de un modo tendencial a través de acciones concertadas, pactadas o negociadas más que por imposición unilateral de mandatos. Lo que ocurre es que estas transformaciones se producen en el marco de una cierta tensión, como ya señalado anteriormente, entre la actuación formal -conforme a las categorías y procedimientos establecidos y regulados por el Derecho- y una actuación informal que cada vez hace más difícil explicar el sistema jurídico únicamente como resultado de su formalización legal.

Aparentemente y frente al modelo propio del Derecho privado liberal que institucionalizó, en cierto modo, la libre elección de modelos de conducta; al posterior desarrollo de importantes sectores del Derecho público que se proponían normativamente más vinculantes, le ha sucedido una mayor flexibilidad de los modelos jurídicos. Lo que ha dado lugar, tal como advierte Ferrari a «un Derecho móvil, disponible, imprevisible, regido por una especie de tácita metarregla que advierte que cualquier regla, durante la interacción puede ser desconocida o modificada $\rangle^{31}$. Tal flexibilidad puede observarse en el ámbito de la administración en cuanto hoy existen mayores márgenes de acción que alcanzan incluso a la negociación o acuerdo sobre los términos de aplicación de la ley, pero también en el Derecho penal, aunque paradójicamente sea el que presente un mayor grado de vinculación. Existe un fenómeno en el ejercicio público de la acción penal que hoy se haya inserto en muchos órdenes jurídicos aunque no esté institucionalizado en todos, el denominado plea bargaining o negociación, que consiste en «el intercambio de concesiones oficiales y un acto de reconocimiento de culpabilidad $\gg^{32}$.

Desde el punto de vista legislativo, además es posible observar cómo no sólo se ha producido un creciente aumento de las normas

\footnotetext{
${ }^{31}$ V. Ferrari, Funciones del Derecho, Madrid, Debate, 1989, trad. J. de Lucas, M. J. Añón, págs. $150-151$.

${ }^{32}$ A. W. Alshuler, «Plea Bargaining and its History», Law and Society Review, vol. 13, pág. 213. Sin embargo, como muestra V. Ferrari, el aspecto más interesante de la «negociación» entre acusado y acusador, desde el punto de vista sociológico, está relacionado con los casos de ejercicio unilateral de la discrecionalidad acusatoria o judicial, como la absolución injustificada o la reducción de las acusaciones y los casos de intercambio de concesiones oficiales y diferentes actos de reconocimiento de culpabilidad, como ofrecer el resarcimiento del daño a la víctima de un delito, dar informaciones o prestar testimonio contra otros sospechosos. Vid. también. C. Menkerl-
} 
de competencia u organización, que como sabemos no responden al esquema tradicional de mandato de carácter general y abstracto y deberes directos hacia los ciudadanos, sino que se regula a través de determinaciones de fines y objetivos, así como de mandatos de búsqueda y encuentro de la solución que en cada caso sea más idónea y adecuada ${ }^{32}$. Cambios que no pueden sino repercutir en el comportamiento de sus destinatarios y especialmente en el de los operadores jurídicos.

Con todo, y como parece evidente, la discrecionalidad no consiste en tener un poder de decisión y realizar una elección, sino en la posibilidad de adoptar una decisión legítima. Por una parte, como hemos advertido, el Derecho crea una específica discrecionalidad, aquella a la que dota de límites y objetivos, Pero la incorporación de situaciones sociales de poder en el marco de una regulación jurídica produce diversos grados de aprobación o confirmación de las relaciones de poder, incluso aun cuando la regulación jurídica a menudo establezca ciertos límites sobre esta legitimación, imponga controles o abandone el reconocimiento de ciertos acontecimiento. Sin embargo, la importancia de esta legitimación interna al sistema depende en gran medida de la existencia de otras razones justificatorias en el mantenimiento de una autoridad como tal.

Por ello, la justificación de los poderes discrecionales ni puede tener sólo un carácter intrasistemático ni puede establecerse de un modo genérico. Precisamente, la importancia que para un orden jurídico

Meadow, «Toward Another View of Legal Negotiation: The Structure of Problem Solving», Los Angeles Law Review, $\mathrm{n}^{\circ}$. 31, 1984, págs. 754-842. Por otra parte, este cambio hacia procesos negociadores en la formulación del Derecho y en el tratamiento de conflictos es uno de los rasgos de identidad del Derecho del Trabajo. A estos efectos puede verse C. L. Alfonso, Proceso de conflicto colectivo. Sistemas alternativos de solución y autonomía colectiva, Valencia, Tirant Lo Blanch, 1993.

${ }^{32}$ L. Parejo, Crisis y renovación en el derecho Público, op. cit. pág. 23. En este sentido sostiene que los instrumentos tradicionales -reglas, mandatos, órdenes- se muestran poco idóneos para articular satisfactoriamente una programación de los objetivos del poder. Por ello se ha acudido, en muchos casos, a evitar los conceptos jurídicos indeterminados y a sustituirlos por fijación de criterios materiales de ponderación y decisión, principios y valores-marco, fines y objetivos de acción, criterios sobre un óptimo social, en definitiva a modelos de referencia que se pretenden suficientes como para orientar una decisión (pág. 48). En el mismo sentido, sobre la toma de decisiones a partir de objetivos políticos y sobre la responsabilidad jurídica y política de ahí derivada, vid. Galligan, Discretionary Powers, op. cit. Además, como muestra M. S. Feldman, «Social Limits to discretion: An Organizational Perpective», en The Uses of Discretion, cuando se ha seguido la técnica de redactar normas absolutamente detalladas con el fin de eliminar la discrecionalidad, se ha producido un efecto contraproducente y la gran complejidad provocada por la profusión de normas ha otorgado mayor libertad de elección al titular de la decisión, págs. 167 y ss. 
tiene la toma de decisiones en cuanto se presume que va dirigida a la realización concreta de objetivos y principios básicos de proyectos político-jurídicos, habrá que determinar su legitimación en cada caso y para ello habrá que interrogarse sobre cómo y por qué se procede a delegar un poder de decisión concreto, cuáles son los criterios o principios que provee tal capacidad de decisión y cómo los realiza. A este respecto, parece importante evitar la presunción en favor de la racionalidad de los imperativos funcionales de tipo burocrático y atender a las razones concretas a partir de las cuales se motiva una decisión. Como se trata por tanto de poner el acento en la esfera de la responsabilidad, la relación entre legitimación y discrecionalidad replantea si una de las funciones del Derecho es la de proveer unos parámetros de legitimación para orientar el comportamiento de funcionarios y ciudadanos. Desde esta perspectiva, suele argumentarse que el poder depositado en instituciones puede transformarse en autoridad en la medida en que los valores y prácticas que son aceptadas o constituyen expectativas en un determinado contexto social son sentidas como legítimas en dicho contexto. La aplicación de este presupuesto a la toma de decisiones por parte de los operadores jurídicos lleva a considerar que esas decisiones deben reforzar o estimular el acuerdo u obtener la conformidad de los destinatarios de la decisión en sentido amplio. Pero desde el punto de vista del fenómeno de toma de decisiones discrecionales se entiende que esta función va más allá de crear relaciones de poder y frecuentemente lo que ocurre es que legitima y regula situaciones de poder existentes. Esta función tiene dos órdenes de proyecciones, de un lado, ofrece al titular del poder discrecional razones adicionales para la acción orientadas a asegurar la legitimación social en la forma en la que ejerce dicho poder. Por otra parte, al sujeto de la decisión y a los terceros les da razones para aceptar el poder de quién tiene esa capacidad discrecional como poder de una autoridad legítima. En este sentido, el Derecho afecta -puesto que validez y efectividad de las normas no son categorías idénticas- a las razones para la acción y a las actitudes de los miembros del grupo social. 
$\triangle$

DOXA 15-16 (1994) 